\title{
Sion, haut-lieu religieux, patriotique et paysager
}

Jean-Pierre Husson

\section{CpenEdition}

\section{Journals}

Édition électronique

URL : https://journals.openedition.org/geohist/525

DOI : 10.4000/geohist.525

ISSN : 2264-2617

Éditeur

Association française de la Revue de géographie historique

\section{Référence électronique}

Jean-Pierre Husson, «Sion, haut-lieu religieux, patriotique et paysager », Revue de géographie historique [En ligne], 16 | 2020, mis en ligne le 20 mai 2020, consulté le 12 juin 2021. URL : http://

journals.openedition.org/geohist/525; DOI : https://doi.org/10.4000/geohist.525

Ce document a été généré automatiquement le 12 juin 2021

\section{cc) (1) $\odot$}

Ce(tte) œuvre est mise à disposition selon les termes de la Licence Creative Commons Attribution -

Pas d'Utilisation Commerciale - Pas de Modification 4.0 International. 


\title{
Sion, haut-lieu religieux, patriotique et paysager
}

\author{
Jean-Pierre Husson
}

1 Le pèlerin voyage et pérégrine. Il est étranger, en rupture avec son quotidien. Ce choix crée une fracture du temps ordinaire, provoquant « à la fois éclatement et élargissement de l'espace ordinaire » (Martin, 1995, p. 213). La route est à la fois son épreuve, son espoir, son but à atteindre. A pied, il emprunte des parcours de traverse pour se déplacer sur des grands chemins ponctués de signes: croix, fontaines miraculeuses, chapelles, églises et lieux où ont été attestés des miracles ${ }^{1}$, où la mansuétude de la Trinité, de la Vierge et des saintes et saints intercesseurs est sollicité par les croyants. Ainsi, en Lorraine existe dans certains lieux la tradition des répits. Un enfant mort-né qui n'a pas pu être baptisé est momentanément ressuscité à la vie pour que l'archange Gabriel puisse intercéder afin qu'il ne soit pasad vitam et ceternam confiné dans les limbes (Martin, 2004). Sion comptait parmi ces lieux.

2 Le sacré est inscrit dans les paysages ruraux anciens et y conserve maintes traces (Peltre, 1995) : itinéraires des rogations, reposoirs, chemins des morts, etc. L'acception classique du pèlerinage trouve ses racines dans l'Ancien Testament. Cette pratique de foi et d'endurance est revivifiée par le récent engouement pour la marche sur les GR, en particulier sur les itinéraires qui conduisent à Saint-Jacques-de-Compostelle. En 1999, la géographe Madeleine Griselin avait suivi ce chemin depuis la Franche-Comté et réalisé des clichés systématiques orientés Est, Nord, Ouest et Sud sur tout le parcours. Un livre a suivi en 2017. En 2013, l'académicien Jean-Christophe Rufin nous a laissé un témoignage au sujet des $850 \mathrm{~km}$ qu'il arpenta sur le Camino del Norte.

3 La référence évoquée est amplement partagée mais convient médiocrement au site qui nous intéresse ici. L'acropole de Sion (Lorraine, 54) domine le Plateau lorrain. Il est dédié à la dévotion mariale qui s'opère ici sans apparition révélée de la Vierge. Le site est d'abord associé à la maison ducale de Lorraine puis à l'ensemble de la nation. Pendant au moins cinq siècles, le politique y croise intimement le fait religieux. L'actuelle trilogie tissée entre le Conseil départemental, récent propriétaire et animateur des lieux, l'Église représentée par un recteur et une congrégation de 
clarisses, enfin tous ceux qui nourrissent la dimension artialisée et magique de ce hautlieu renouvelle les synergies créées autour du site dont la fréquentation annuelle est estimée à 120000 personnes. Après 1871, le duo formé par l'Eglise et le pouvoir est conforté par la défaite et la volonté de redressement qui s'ensuit. Ce duo grandit dans le contexte de la Nation meurtrie, amputée par le traité de Francfort. Si l'on se reporte plus loin dans le temps, le lieu attira toujours des foules inquiètes, traumatisées par les guerres puis portées par un nationalisme qui espérait en la revanche.

4 Promontoire emblématique formé par une vaste butte témoin arquée située au cœur des riches terroirs à blé du Saintois, la butte de Sion s'explique par la flexure qui imprime ici la dalle solidement armée des calcaires coquilliers. La résistance dictée par l'avantage de la couche dure dans le binôme avec les marnes, la flexure et la relative activité des ruisseaux cataclinaux expliquent cette proue. La carte de Vézelise Est 3316 montre un commandement de $176 \mathrm{~m}$ entre la basilique (497 m.) et le village de Praye juché à son pied $(321 \mathrm{~m}$.). Le site était desservi par la ligne Nancy-Merrey, ouverte en 1880, actuellement fermée. Nous sommes à $30 \mathrm{~km}$ au sud de Nancy. Cette proximité explique la permanence de la dévotion exprimée par la capitale ducale, en particulier celle de ses ducs René II, Henri II de Lorraine, Charles IV ${ }^{2}$. En 1661, le duc Charles IV (1604-1675) (Fulaine, 1997) dispose de la restitution de ses duchés mais est contraint à un nouvel exil en 1670. A cette date, Louis XIV occupe à nouveau la Lorraine pour préparer la guerre contre les Provinces-Unies. Anticipant ce fait, le duc avait choisi N.D. de Sion comme « souveraine de la couronne et de tous les sujets lorrains». Par ce geste, il croise la destinée du lieu de pèlerinage avec l'identification de la dynastie ducale. En 1663, il accroit l'aura des lieux en dédiant à N.-D. de Sion la relique d'une sainte épine de la crucifixion. Dès 1627, il accueille les Tiercelins sur le site. Ils furent présents jusqu'à la Révolution (Andriot, 2006). Cet éclairage sur le trouble XVII ${ }^{\mathrm{e}}$ siècle traduit par les effets désastreux de la Guerre de Trente Ans désigne un temps de très lente convalescence où la foi s'exprime de façon intense (Martin, 2002).

5 La compréhension, la promotion et la pérennité du site relèvent de données différentes de ce qui anime Compostelle, Lourdes, N.-D. de La Salette, Fatima, etc. Exception faite de la période 1871-1973 (le 9 septembre 1973 clôture les manifestations de la réconciliation franco-allemande), le pèlerinage reste à dimension régionale. L'approche géo rétrospective qui croise des temps longs, parfois rompus, accélérés, voire des temps d'expectative par rapport au devenir d'un site sied à l'étude de Sion. Ici, d'autres leviers que les miracles et visions ont été mobilisés sur une frise du temps longue incorporant heurts et malheurs des guerres. Le pèlerinage s'est renouvelé sur plus d'un millénaire d'existence. Trois temps forts se succèdent.

6 En premier, la dévotion accompagne tous les drames épidémiologiques (grande peste de 1423 , diffusion du choléra en 1854) et géopolitiques qui parsèment l'histoire des duchés. Positionnée pour son malheur en situation d'entre-deux (Jalabert, 2014), la Lorraine est marge, espace de conflit depuis le traité de Verdun de 843. L'annexion des Trois-Evêchés confirmée par la paix de Westphalie (1648) conforte cette faiblesse. Le mince statut quo proposé à Léopold avec la paix de Ryswick (1697) laisse perdurer un espace vulnérable.

7 En second, l'alchimie établie entre la nation et la foi pour ériger Sion en haut-lieu. Pour B. Debarbieux (1995, p. 102), le haut-lieu « (...) est spécifié par son caractère sacré au sein d'une cosmogonie-entendons le ciel structuré par les divinités associées aux astres par opposition au chaos- d'essence religieuse ( ), ce qui procède par discontinuité et 
par désolidarisation avec l'espace profane». Doublement porté par la piété mariale protectrice et par le patriotisme meurtri, le site marial de Sion prospère pendant toute la durée de la Troisième République, voire plus tard dans le contexte de la réconciliation franco-allemande. Dès 1870, le pape Pie IX accorde à la Vierge de Sion le privilège d'être couronnée. En 1873, après le départ des dernières troupes d'occupation et le paiement d'un tribut de 5 milliards de francs or, une grande manifestation est organisée pour célébrer ce couronnement de la Vierge.

Enfin, l'actuel change la donne mais conserve à Sion son capital de fréquentation. En 2000 , le site est acheté par le Conseil départemental. Peu après se déclare un vaste incendie (2003) exigeant quatre années de travaux avant de remonter au sommet du clocher la Vierge monumentale ( $7 \mathrm{~m}$. de haut, 8 tonnes) sortie en 1856 des fonderies de Tusey (Meuse). Le CD 54 a réaménagé les bâtiments conventuels pour y créer une Cité du paysage inaugurée en 2015. De nouvelles questions et solutions sont expérimentées pour faire vivre ce haut-lieu, y faire évoluer les récits en lien avec sa trajectoire territoriale (Sgard, 2008). Il s'agit à la fois de s'inscrire en continuité et en rupture avec les passés empilés évoqués. L'objectif est de s'ancrer dans la légitimation de l'Histoire, voire des histoires pour passer d'une politique mémorielle à une priorité accordée aux approches patrimoniales. Ceci débute dès 1936 avec le classement de 130 hectares de pelouses sèches situées sur la commune de Vaudémont. Cet acte est le point de départ d'une reconnaissance qui passe aujourd'hui par un site Natura 2000 qui concerne 12 communes.

9 Avec ses outils et méthodes, l'approche de géographie historique sied bien pour tisser $\mathrm{du}$ lien entre les temps qui firent vibrer, évoluer voire sommeiller Sion et la colline inspirée. Cette démarche s'effectue autour de plusieurs questions qui s'articulent, se succèdent, se complètent et s'entrechoquent. Approches synchroniques de ce qui se passe en même temps et prise en compte des dynamiques du déroulé du temps éclairent d'un jour différent les lieux. Ces derniers évoluent dans des alchimies successives. Ils sont réunis dans une matrice spatio-temporelle qui place l'espace en abscisse et le temps en ordonnée. Ce choix signifie que l'espace prime sur le déroulé du temps et n'oublie pas de prendre en compte la dimension prospective. Ce propos est en résonnance avec la pensée de J.-R. Pitte (2005) pour qui la géographie historique est « un terreau pour l'imagination d'aujourd'hui ».

10 Sion est réputé être un lieu où le surnaturel tutoie la beauté des panoramas. Cet espace de mémoire se prête à la fois à la quête des racines, à la connivence avec le sacré, à la recherche de symboles (Louis, 1990). Depuis 1913, l'ambiance particulière qui s'en dégage reste sous l'ombre portée de l'œuvre de Maurice Barrès (1862-1923). Peu avant son décès, l'académicien écrivait de façon prémonitoire « On ne pourra plus aller à Sion sans m'y trouver » (Mes cahiers, tome XIV, p. 41). Cette sorte de magie est relayée par maintes productions artistiques et littéraires. Elles prennent pour inspiration le site marial élargi à la lanterne des Morts inaugurée en 1928 par le président Poincaré et le maréchal Lyautey (Surdel, 2006) et encore les ruines du château de Vaudémont. Ces trois points occupent les trois sommets de la butte témoin qui culmine au signal de Vaudémont. Il est nécessaire de partir de la présentation de cette proue pour en éclairer la dimension exceptionnelle, la poésie qui s'en dégage. L'aménité de la colline inspirée est largement véhiculée par le filtre de l'artialisation (Husson, 2019). Ce préalable acquis, la démarche de géographie historique privilégie l'approche des temps forts répercutés sur le site pour comprendre Sion. Il s'agit d'éclairer les relations 
à géométrie variable tissées entre, d'une part la continuité exprimée pour la piété mariale et d'autre part les drames, tensions, accélérations des conflits politiques et militaires vécus in situ en Lorraine ou à l'échelle de la Nation et de l'Europe. La filiation entre ces deux données fit vivre et assez souvent vibrer le site, par exemple quand monseigneur Turinaz, évêque de Nancy de 1882 à son décès en 1918 venait exhorter les foules de discours patriotiques. L'alchimie secrétée entre le pouvoir et l'Eglise dura tant que l'ennemi fut visible, identifié, menaçant. Les lois de séparation de 1905 modifièrent peu ce constat. Aujourd'hui, ce contexte est très éloigné de la réalité de nos vies majoritairement matérialistes. Nous sommes engagés dans une période nouvelle où le couple formé par la foi et le nationalisme s'est, du moins chez nous, totalement délité, à peine réveillé par des émotions collectives, la dernière pouvant être l'incendie de Notre-Dame de Paris (2019). Sion a dû chercher d'autres chemins pour vivre. Ainsi, la Cité du paysage, la promotion du commerce équitable cohabitent avec l'activité pèlerine. L'écologie des paysages porte désormais le lieu et forme une nouvelle couche d'histoire qui s'accommode bien des héritages précédents, sauf probablement ce qui a été légué par la période 1871-1945.

\section{Un lieu exceptionnel où souffle l'esprit}

11 La colline de Sion est géomorphologiquement une grosse butte témoin placée en avant de la cote de Moselle. Dans un entretien daté du 25 août 2013, l'écrivain Philippe Claudel de l'Académie Goncourt décrit ainsi ce monument : "Quand on s'approche de loin, on commence à apercevoir dans la campagne lorraine une sorte d'énorme bateau, de barge renversée. La colline de Sion est un lieu qui compte toujours autant». Ce lieu accumule plusieurs millénaires de mémoires archéologiques empilées, stratifiées. Pour L. Olivier (2006), la lecture archéologique du site donne un supplément de sens à ce site si riche en histoire. C'est en particulier le temps fort du Bronze final ( $\mathrm{X}^{\mathrm{e}}$ siècle avant J.-C.) et celui de la prospérité de la ville fortifiée par les Leuques (II siècle avant J.-C.).

Faute de bénéficier d'un balayage LIDaR de l'oppidum, nous disposons d'un inventaire systématique effectué en 1985. Il couvre 750 ha. et révèle 80 sites répartis du Néolithique au Moyen Age. Elargi à un périmètre de $10 \mathrm{~km}$, l'inventaire s'élève à 700 sites. Le lieu est aussi salué par tous les artistes, en particulier le graveur André Jacquemin qui contribua fortement à soigner la dimension artialisée du site : «Tout est puissance dans ce haut-lieu ( ), l'on savoure le pays du Xaintois (le Saintois s'orthographie Xaintois quand cette petite région déborde sur le département des Vosges) qui garde encore sa vieille âme de jadis » (Jacquemin, 1962). Ilot élevé placé entre la côte de Moselle et, plus à l'Est celle de l'Infralias, Sion est porté par le fait religieux depuis la préhistoire. Le site profite d'une filiation débutée avec les cultes gaulois et antiques: Rosmerta, déesse de la fécondité puis Mercure, guide des voyageurs et des commerçants travaillant sur de longues distances. Proche du site apollinien (karst déifié) de Grand et placé en retrait du grand axe Langres-Trêves servant à la pénétration des cultes nouveaux apportés par les légions, Sion sombre pendant les temps obscurs mais renait dès le $\mathrm{XI}^{\mathrm{e}}$ siècle. A Sion, la verticalité du site favorise l'approche paysagère. C'est une " endroit où le ciel et la terre se touchent " (Corajoud, 1995), où le panorama à $360^{\circ}$ prend tout son sens.

13 La colline de Sion prend l'aspect d'une vaste proue arquée dessinée en forme de fer à cheval. Cette bizarrerie a marqué les esprits depuis la nuit des temps et conforte l'idée 
de haut-lieu. Cette forme est née de la résistance du binôme calcaire fortement charpenté. Né à l'ère jurassique de l'empilement des dépôts marins, il est à l'origine de la fossilisation des encrines (étoiles de Sion). Les couches sont à la fois inclinées, localement flexurées, affectées de failles; ce qui éclaire l'isolement du lieu par rapport à la côte et encore la capacité à résister à l'érosion régressive. Localement, les effets érosifs dégagent la butte témoin bajocienne dominant les marnes du Toarcien et du Domérien qui supportent les riches terres à blé du Saintois. La butte de Sion appartient à une suite de sommets tous très originaux : la butte de Pulney, le bois de Curel, la calotte du Bois d'Anon, le mont Thélod.

Document 1 : La colline de Sion, mise en parallèle de l'extrait de la carte géologique et de l'image satellitaire du lieu

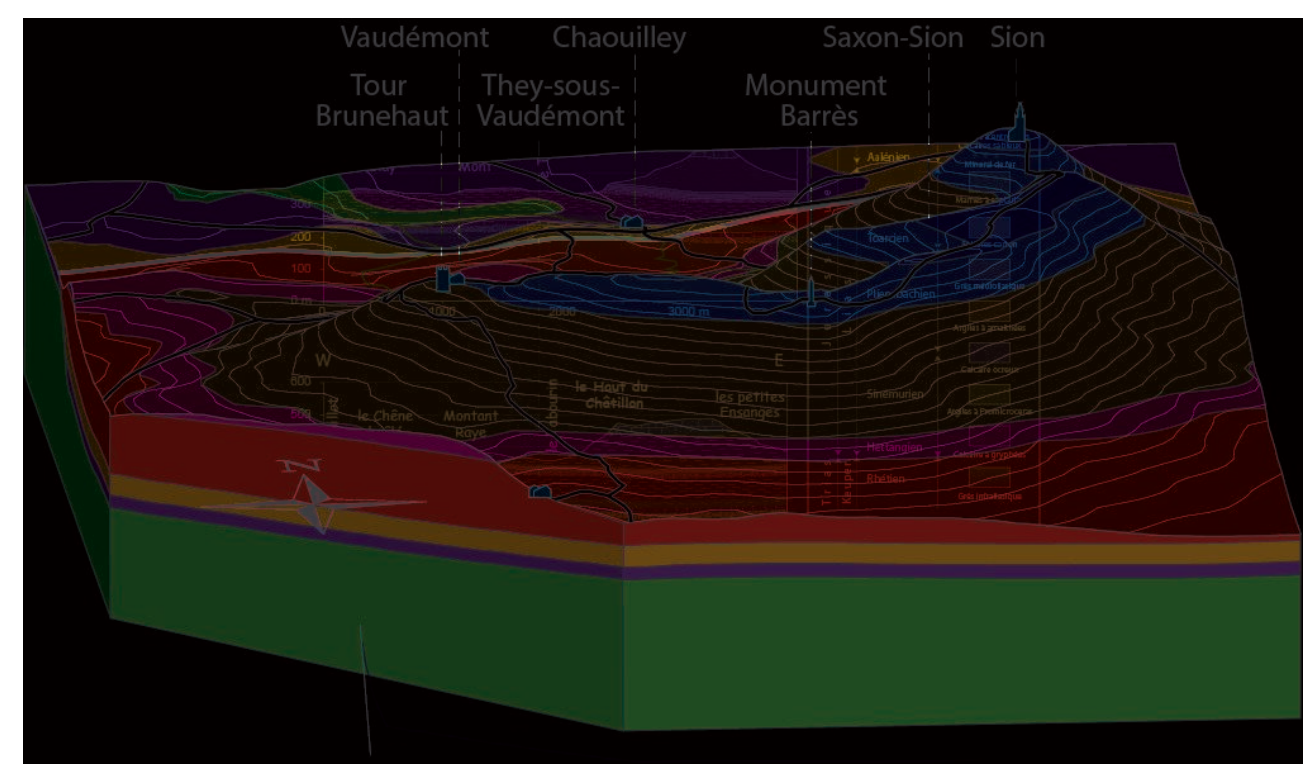

Source : Le Roux Jacques, Harmand Dominique, 2011, Cadre géomorphologique de la colline de Sion In Giuliato Gérard, 2011, Autour des comtes de Vaudémont, lieux et symboles du pouvoir princier. Nancy, PUN, p. 19-51.

14 La forme de proue crée une topographie particulière qui a toujours retenu l'attention des peintres et photographes, en particulier Bertho et Conésa (2017, p. 240) pour qui la topographie est une valeur créative à part entière. La foi aime s'exprimer dans le sublime, les belvédères. Venté, givré en hiver, en permanence balayé par les flux d'ouest, Sion est aussi intensément fleuri pendant la courte et incertaine période de l'éclosion des fleurs blanches des mirabelliers, ce qui est à rapprocher de sakura, la floraison des cerisiers au Japon; un événement national attendu, fugace, capricieux. Les vergers anciens (haute tige) ou récents (arbres courts taillés en gobelets) drapent ses flancs aux bonnes expositions et ont alterné avec les vignes dont la présence est ici relictuelle. Au contraire, la carte militaire établie par les Naudin entre 1728 et 1739 à l'échelle approximative du $1 / 28800^{\circ}$ (Wagner, 2003) ou la première version de la carte dite d'Etat-major signalent le versant Sud (They-sous-Vaudémont) et Est (Praye) couverts par cette culture.

La production de mirabelle est une autre représentation de ce territoire et les logos ne manquent pas d'associer la silhouette de la colline et les fruits couleur or (Wadier, 1991). Ils sont transformés en confitures, jus, fruits séchés, tartes, et autres gâteaux, sans oublier la "goutte " (alcool blanc tirant à $\left.52^{\circ}\right)$. Ces produits associent la colline à 
l'image de la gastronomie locale. Le drapé de vergers n'est pas continu. Sur les endroits mal exposés, il est relayé par la friche qui évolue souvent en ourlets boisés à surveiller pour garder ouverts les sites panoramiques. Parfois, la pente est conservée en catena ou chaine de sols formés d'éboulis. Conservé sur une ligne de partage des eaux secondaire entre le Madon et le Brénon, Sion est un môle lithologiquement résistant grâce à l'armature des calcaires polypiers entrés dans la légende et le récit du site. Lors de l'inauguration de 2015, le CD 54 avait mobilisé des troupes d'acteurs pour évoquer la magie des lieux. L'un d'eux, tout de jaune vêtu incarnait le calcaire à polypiers. Avançant son grand âge, il donnait des étoiles de Sion un récit poétique mais qui différait de la tradition des pèlerins retenant une pluie d'étoiles offerte par la Vierge. Les lys de mer (encrines) fossilisés dessinent des minuscules étoiles qui ont fait la joie de générations de «fouilleurs ». Dans le contexte du Choc pétrolier, le professeur P.-L. Maubeuge tenta même l'extraction d'hydrocarbures trouvés dans les grès, à environ $500 \mathrm{~m}$ de profondeur, au pied de la colline (Forcelles-Saint-Gorgon, Chaouilley).

La simplicité de l'ordonnance des lignes du paysage a ému tant de peintres et écrivains ! Elle participe au renouvellement du site initialement associé aux familles ducales (Alsace, Anjou, Vaudémont puis Habsbourg-Lorraine suite au mariage de François III avec Marie-Thérèse d'Autriche en 1736). Sion ne se résume pas à un lieu de pèlerinage. C'est un endroit où la géologie, la mise en scène des paysages, l'histoire ducale et la dévotion à la Vierge ont créé un curieux assemblage, une sorte d'attelage qui a permis de traverser les siècles.

\section{Document 2 : Sion vu depuis Chaouilley}

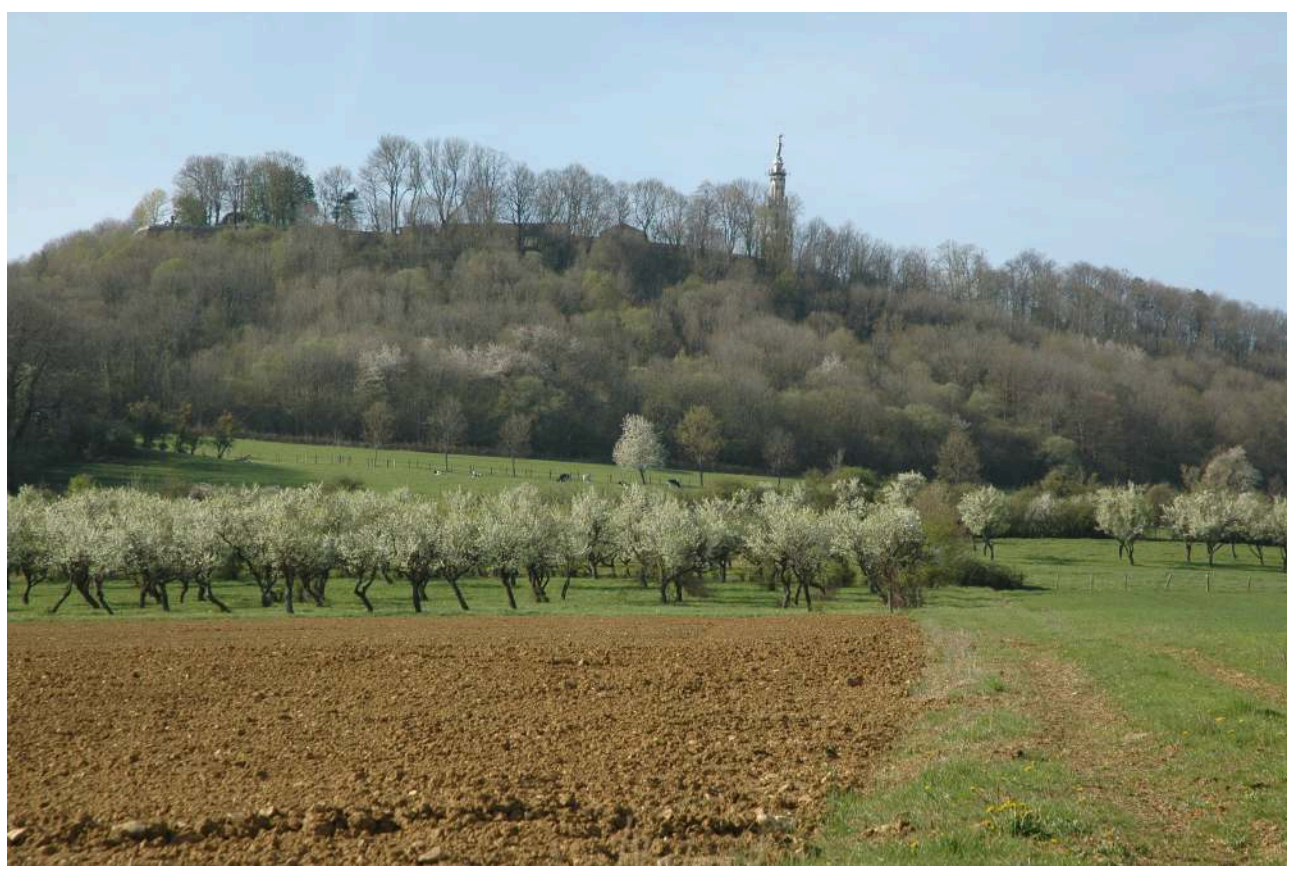

La colline à la forme arquée domine de $180 \mathrm{~m}$ la dépression du Saintois. Le paysage a beaucoup changé depuis un siècle. Les lambeaux forestiers sommitaux ont payé un lourd tribut au passage de la tempête Lothar (1999). Les pentes sont gagnées par de denses ourlets de friches. Les jeunes vergers taillés en gobelet occupent le bas des versants. Les vieux vergers à haute tiges s'éclipsent, la vigne a disparu. Le clocher de la basilique rehaussé d'une monumentale vierge domine l'ensemble et sert de très loin de signal.

Source : cliché CERPA, D. Brion, 2010. 


\section{Epreuves et drames créent, confortent le rayonnement de Sion}

17 Avant la phase de forte déchristianisation qui affecte le pays dès la fin des Trente Glorieuses, Sion a entassé les mémoires successives de passés glorieux ou tragiques. Le site traverse le temps et ses furies. Les pèlerinages de la fête de l'Assomption) ont été très fréquentés. Le lieu contribue à créer et entretenir une identité lorraine qui, aujourd'hui a perdu beaucoup de son sens, jusqu'à fondre ce territoire dans une nouvelle entité administrative appelée Grand Est.

\section{A. Un sanctuaire lorrain}

Dès la Renaissance, la renommée de Sion est établie. Le duc René (1451-1519) associe l'avenir de sa dynastie à la protection de la vierge de N.-D. de Sion après avoir brisé le rêve conquérant du Téméraire tué devant Nancy en 1477. Sa sœur Marguerite de Lorraine (1463-1521) est statufiée dans un arbre creux planté au pied de la basilique. A la fin de sa vie, elle entre chez les clarisses et devient bienheureuse. Ce signe de la papauté rehausse la collusion tissée entre le pouvoir ducal et le culte de la Vierge à Sion. Henri II et son épouse Marguerite de Gonzague poursuivent les efforts entrepris, entretiennent ce lien de piété mariale. En 1622, cette princesse (1591-1632) née à Mantoue fit ériger une croix localisée en bord du chemin reliant Sion à Vaudémont, avec pour signe d'identification au lieu, les armes des deux familles sculptées sur le tablier du monument. Bref, à chaque moment heureux ou tragique, la dévotion s'amplifie, y compris s'il s'agit de trouver des parades à des dérèglements climatiques, avec par exemple une chute de la température moyenne estimée à $1,3^{\circ}$ sur la période 1690-1710. Encore à la vieille de la Révolution, lors de la grande sécheresse de juin 1784, 32 processions furent organisées pour demander l'intercession de la Vierge de Sion (Martin, 1995).

Le très troublé XVII ${ }^{e}$ siècle qualifié de tragique par l'historien Pierre Goubert est un temps fort pour souder le couple formé par la famille ducale et N.-D. de Sion. D'abord se pose un problème de légitimité pour lever la filiation entre le dernier Anjou et son successeur Vaudémont issu d'une branche ducale cadette. Pour cela, on retrouve à Sion un acte de René II daté de 1504 instituant le principe de la transmission de la couronne ducale par les hommes. Nous sommes en 1633, à l'aube de l'entrée de la Lorraine dans le malström de la Guerre de Trente Ans. L'artifice est bénéfique mais ne suffit pas pour assoir le pouvoir de Charles IV, certes malheureux diplomate mais surtout prince exilé devant faire face aux pressions exercées par ses puissants voisins. Charles $\mathrm{V}$, puis à son retour d'exil Léopold se placèrent également sous la protection de la Vierge. Même Stanislas poursuivit cette attention. La famille royale de Habsbourg-Lorraine demeura fidèle à N.-D. de Sion et même Otto de Habsbourg vint ici en pèlerinage.

\section{B. Un sanctuaire national pour un pays meurtri}

Dans son livre sur L'Esprit lorrain paru en 1991, le contemporanéiste Pierre Barral souligne le paradoxe d'un patriotisme français porté par les Alérions et plus encore associé à la référence à la croix de Lorraine (Le Tacon, 2012). Sion, c'est tout cela; le télescopage entre ce qui résulte de l'histoire ducale et le destin national d'un pays 
amputé après avoir vécu plusieurs siècles de conquête ou de résistance à ses adversaires pour ne pas être submergé , si l'on fait exception de l'épisode des Cent Jours. Le traumatisme né de la défaite de 1871 profite à Sion. A la fois patriotique et religieuse, la manifestation du premier « Po Tojo » (doc. 1) réactive le pèlerinage hissé au rang d'audience nationale. La foi exprimée sur la colline est indissociable de l'esprit de revanche à entretenir autour de la bannière de la ville de Metz qui est conservée sur ce lieu. Dans une France ultramontaine qualifiée de "fille aînée de l'Eglise» (oraison du R.P. Lacordaire à N.-D. de Paris le 14 février 1841), récupérer à sa cause l'intercession de la Vierge et des saints est une réponse au Reich dominé par la Prusse luthérienne. En 1890, monseigneur Turinaz associe Jeanne d'Arc et N.-D. de Sion dans une même défense de la nation.

Document 2 : Principaux épisodes qui font vibrer Sion à l'époque contemporaine (1873-1973)

\begin{tabular}{|l|l|}
\hline Date & Evénement \\
\hline $\begin{array}{l}10 \text { sept. } \\
1873\end{array}$ & $\begin{array}{l}\text { Dépôt d'une croix de Lorraine brisée avec la mention «Ce n'ame po tojo! »*et } \\
\text { couronnement de la Vierge. Après 1918, la brisure fut masquée par une palmette d'or. }\end{array}$ \\
\hline 1913 & $\begin{array}{l}\text { Publication du livre «La colline inspirée ». M. Barrès apporte ainsi au site une } \\
\text { aura artistique intense }\end{array}$ \\
\hline $\begin{array}{l}24 \quad \text { juin } \\
1920\end{array}$ & Pèlerinage du second « Po tojo »pour célébrer la victoire \\
\hline $\begin{array}{l}23 \text { sept. } \\
1928\end{array}$ & Inauguration de la Lanterne des Morts par le président Poincaré et le maréchal Lyautey \\
\hline $\begin{array}{l}\text { sept. } \\
1946\end{array}$ & Pèlerinage du troisième « Po tojo » en présence de Jean de Lattre de Tassigny \\
\hline $\begin{array}{l}1973 \\
\text { sept. }\end{array}$ & Cérémonie de réconciliation franco-allemande \\
\hline
\end{tabular}

*Signifie « ce n'est pas pour toujours » en patois du Saulnois, territoire francophone annexé en 1871.

21 En 1913, Maurice Barrès, à la fois élu académicien et député en 1906, fait paraître « $L a$ colline inspirée ». Ce livre qui eut un grand retentissement transfigure le site, modifie à jamais son image et même son appellatif. Dès l'introduction, M. Barrès classe Sion parmi «les lieux qui tirent l'âme de sa léthargie, les lieux enveloppées, baignés de mystère, élus de toute éternité pour être le siège de l'émotion religieuse ». Dans son testament (1893), Jules Ferry avait évoqué la ligne bleue des Vosges, une expression transformée en synecdoque pour évoquer autrement qu'à Sion la mémoire des provinces perdues. Pour J.-F. Thull (2006), Barrès a fixé dans les imaginaires l'appellation du lieu. Avant lui, on parlait de «montagne" ou de "côte de Sion". L'écrivain fait entrer le processus d'artialisation dans la compréhension de ce haut-lieu voué au sacré dans sa forme particulière, celle de la blessure du nationalisme meurtri. Le document 3 tente de traduire cet impact en un triptyque où trois types de relations interagissent. Le sacré n'est plus résumé à l'identité lorraine mais porté par l'ensemble de la Nation. La Grande Guerre et la Victoire créent les conditions d'un pic de légitimité 
et de renom pour Sion. Ce stade de reconnaissance ne sera plus jamais atteint par la suite.

Document 3 : Triptyque de convergences nées à partir de l'œuvre la colline inspirée (1913)

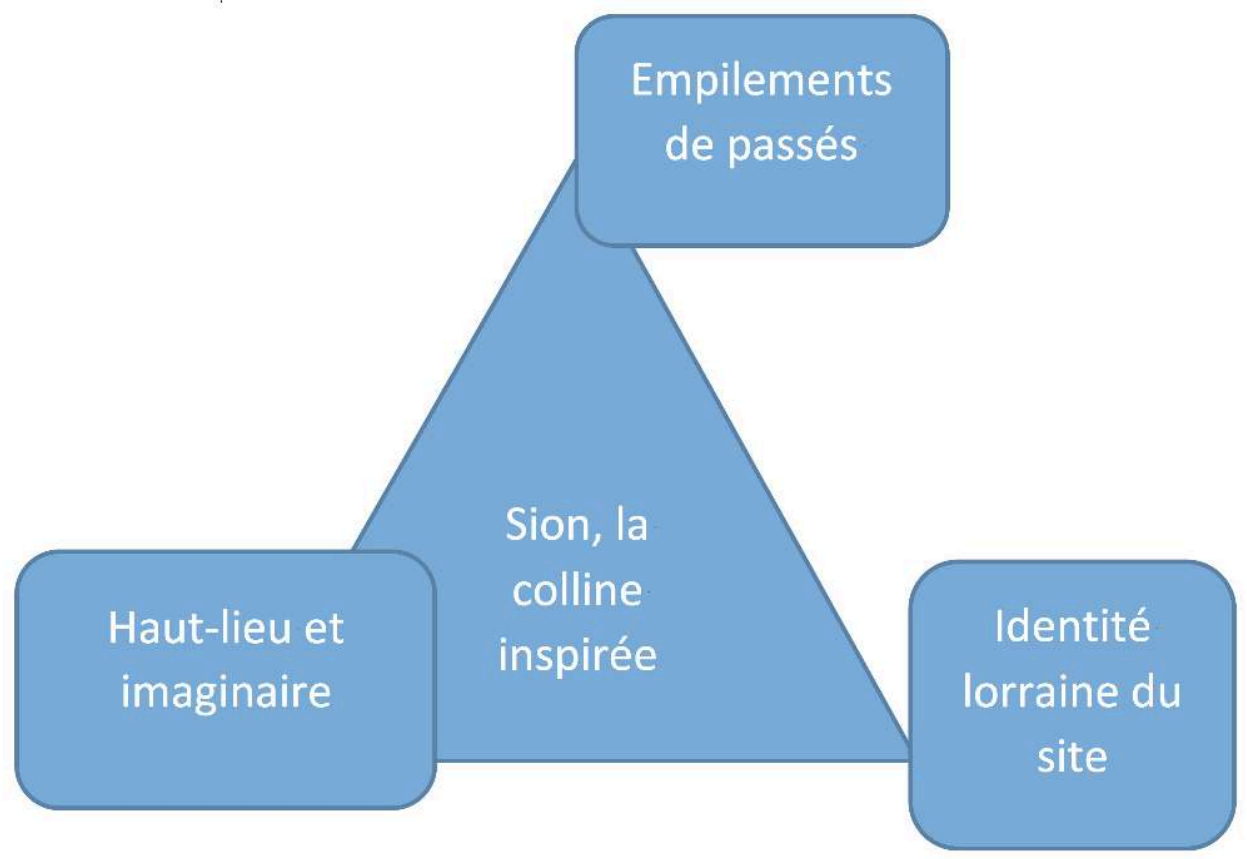

La Grande Guerre a couturé, lacéré la Lorraine où s'enfonce durablement un long bandeau du front, une cicatrice aujourd'hui révélée par les balayages LIDaR. Sion est en-deçà du front mais le site vibre au rythme des manifestations patriotiques qui y sont organisées. La ferveur nationale structure la fête de la Victoire sous l'aile bienveillante de la « douce et bonne mère ». En 1920, suite au second «Po Tojo!», les murs de l'église sont couverts d'ex-voto. En 1933, le Pape accorde le titre insigne de basilique mineure à N.-D. de Sion. Peu avant, en 1928, l'inauguration de la Lanterne des Morts par le président Poincaré et le maréchal Lyautey marque un double changement dans la géographie locale de la dévotion. S'opère un déplacement de la ferveur sur un troisième point de la colline. Jusqu'ici, le dialogue se faisait seulement entre la pieuse Sion et Vaudémont, certes chevaleresque mais démantelée. La construction d'un monument dédié à Barrès et à son œuvre littéraire sort du champ religieux. La Lanterne des Morts est inspirée de celle qui fut érigée à Feniaux (Charente Maritime) au XII siècle. Elle est l'œuvre de l'architecte Achille Duchène (Surdel, 2006). D'importants travaux très récents viennent d'être entrepris pour que le site soit accessible en fauteuil roulant. La canalisation du public sur des chemins tracés facilite le respect du classement en ENS des pelouses sèches périphériques. 


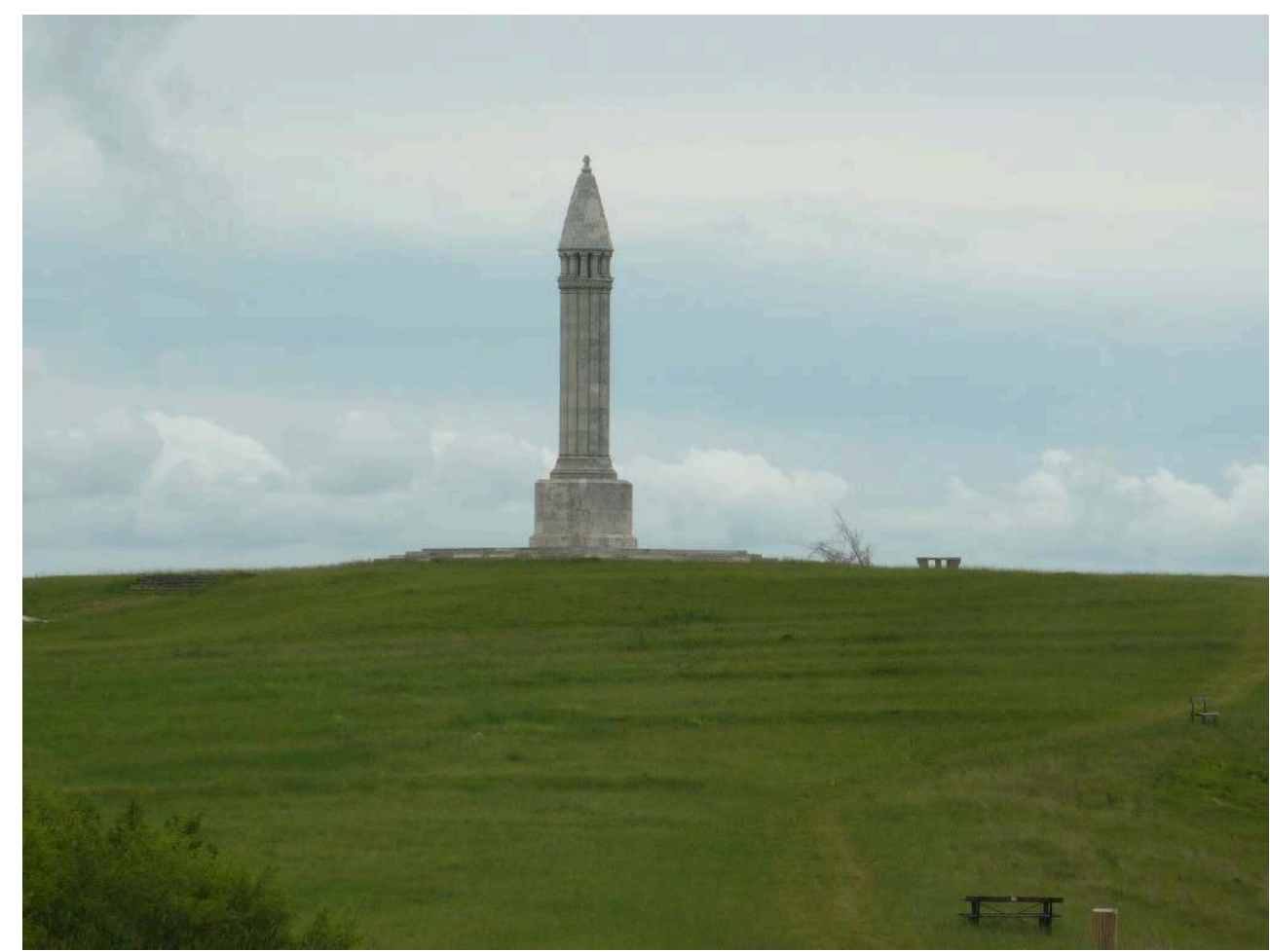

Sous un ciel gris, tourmenté, le cliché s'organise en deux plans. D'abord des palimpsestes agraires anciens qui signalent que les rendzines minces ont été dans des temps très reculés des terres faciles à travailler. Au second plan, le monument dédié à Barrès et la table d'orientation. La verdeur des couleurs est trompeuse. La pelouse sèche, xérique, classée en ENS est souvent grillée par le soleil et le passage répété des vents.

Source : cliché J.-P. Husson, 2017

Le troisième «Po Tojo » a lieu 73 ans après le premier, sous le haut patronage de Jean de Lattre de Tassigny, maréchal de France à titre posthume après avoir incarné le ralliement à de Gaulle et l'épopée de l'armée de libération Rhin et Danube. Cette immense manifestation réunit 80000 personnes. C'est un des sommets dans la ferveur du couple faisant vibrer de consœur la foi et l'amour de la nation. Après ce temps exceptionnel, progressivement prier à Sion rentre davantage dans la sphère de la démarche privée que dans l'expression de la dévotion collective. Ceci est bien perçu en étudiant les cahiers de pèlerinages conservés par les Oblats (Martin, 2006). La prière est surtout demande, y compris celle de la compréhension d'une situation personnelle délicate (deuil, pauvreté, etc.). Ainsi, longtemps allèrent se marier en catimini à Sion les couples qui attendaient un enfant. Une autre image fut celle du rapprochement francoallemand officiellement célébré en 1973, cent ans après le premier «Po Tojo ", avec la pose d'une simple mention gravée « Réconciliation».

\section{Dernière bifurcation pour Sion, un site entre artialisation, naturalité et foi}

La colline doit évoluer pour perdurer, demeurer dans l'événementiel, voire l'affichage médiatique. La basilique, l'esplanade où se font les offices des grandes cérémonies (15 août par exemple), le petit et grand tour de procession ne sont plus les seuls objets 
servant d'accroche pour valoriser le site qui doit se nourrir d'un récit à réinventer. L'alchimie précédente reliait la création artistique et l'artialisation du site, la foi, les pèlerinages et les processions, enfin la mobilisation de l'histoire et les commémorations. Désormais, ce récit et la mise en scène attendue changent, pouvant même prendre une dimension sportive originale. Le «Trail de la colline » est organisée à l'automne et se décline en deux circuits de 15 et 31 kilomètres. Les randonneurs du Saintois ont fléché sur le site de la Colline trois itinéraires (cercle vert, bleu, rouge) et fournissent d'abondantes explications sur leur site http:// www.lesrandonneursdusaintois (onglet nos circuits). Désormais, les lieux s'articulent à géométrie variable autour de quatre éléments qui se complètent, voire s'épaulent pour entretenir une certaine magie. Il s'agit de la basilique, du donjon ( $\mathrm{XI}^{\mathrm{e}}$ siècle) de l'éperon barré de Vaudémont appelé tour Brunehaut (reine d'Austrasie, vers 547-613), de la lanterne des Morts.

Ajoutons les pelouses sèches et le drapé des vergers qui ont largement investi les pentes après la crise phylloxérique. Le site s'est beaucoup laïcisé. L'idée est d'y développer un nouveau souffle avec pour sujet l'interprétation des paysages durables, la fugacité des images de paysages éphémères créées par la variabilité du climat, en profitant des inversions thermiques qui sont courantes. Localement, le ciel peut être d'un bleu azur alors que le Saintois reste noyé dans la brume. A Sion, l'air est agité, pétri par les vents. La neige persiste en hiver. Les anticyclones peuvent dessiner localement des paysages hivernaux taillés dans la carapace du verglas et du givre. Cette fugacité des changements continue à inspirer les artistes à la suite de Victor Guillaume qui résida à Vaudémont, Camille Hilaire, Jacques Hallez, P.E. Colin, Géo Condé et André Jacquemin. Elle anime aussi la pensée des amateurs de parapente qui ont investi les lieux et se lancent dans les airs à partir de Vaudémont pour renouveler le rêve d'Icare, avec des vents forts, des courants ascendants. 


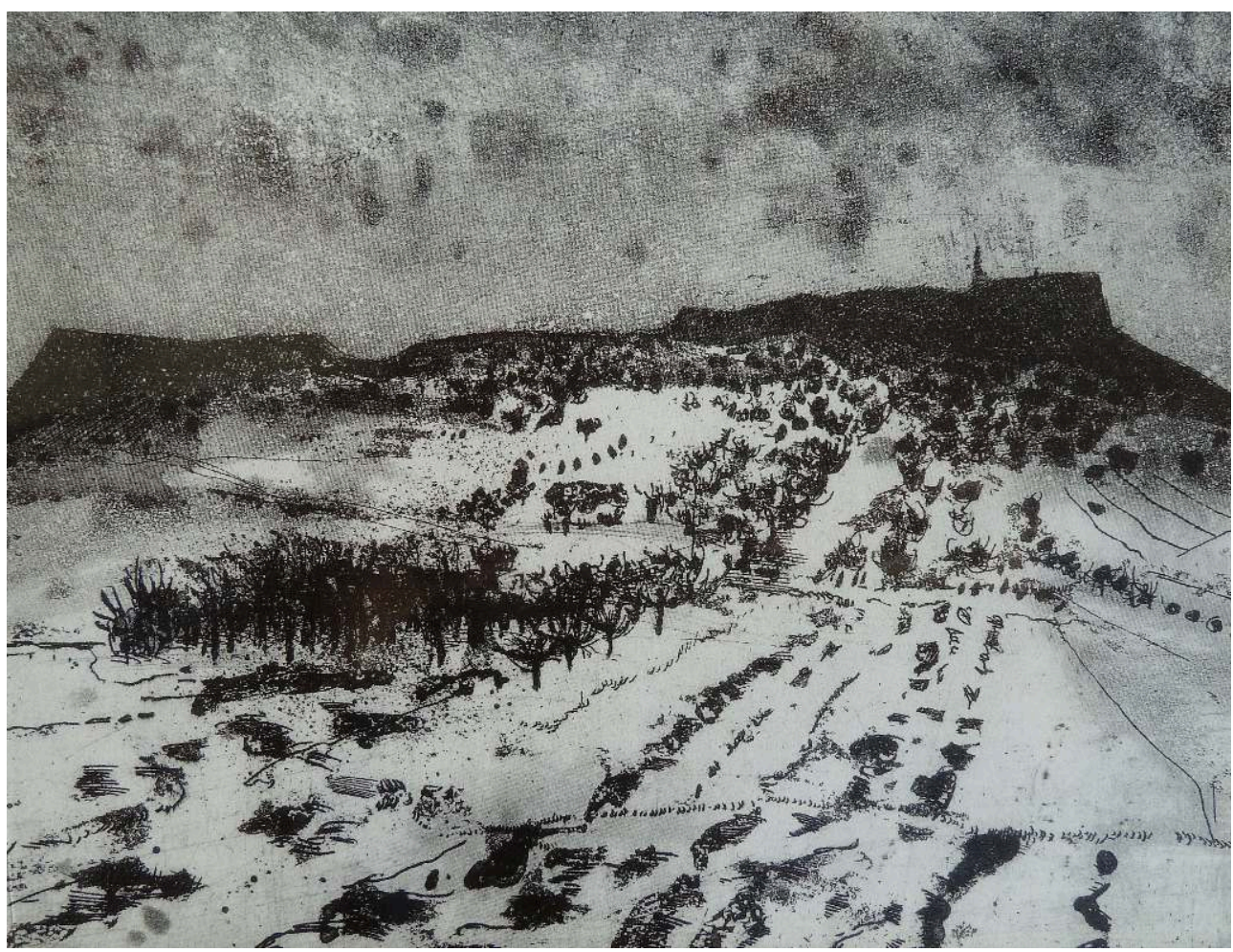

Sur cette gravure de Jacques Hallez, la pointe sèche traduit le ciel intensément gris et empli de neige. Le site résumé à la carapace calcaire est surligné de noir, avec en proue la basilique. Des vergers dégringolent des pentes encore largement ouvertes, striées de sillons et de rideaux de haies. L'auteur remercie l'artiste pour cette œuvre déjà publiée dans la revue Le Pays lorrain, $\mathrm{n}^{\circ}$ spécial 2004 du centenaire de cette publication. L'artiste rejoint très certainement madame Françoise Hervé, inspectrice régionale des sites qui affectionne ce lieu et évoque à son sujet « un bateau perdu dans le brouillard ».

\section{Jalon écologique et paysager}

27 L'imagination a toujours été au rendez-vous pour qui fréquente, est en connivence avec la colline inspirée. Endroit où la mémoire est ressource, Sion négocie un tournant pour devenir un tiers lieu, ce qui peut être défini comme espace de projet, de rencontre et de formation. C'était très probablement ce qui animait Michel Dinet (1948-2014), président du Conseil Général, concepteur du pays de Colombey-les-Belles et premier président de l'UNADEL (Union Nationale des Acteurs et des structures de Développement Local) quand il s'est penché sur Sion et qu'il a encouragé l'achat du foncier. In situ, il voulut concrétiser un projet pédagogique et artistique invitant à réfléchir à diverses formes de transition (dont la transition énergétique). La Cité des Paysages est un équipement culturel du CD 54 qui a investi $800 \mathrm{~m}^{2}$ de locaux situés dans les anciens bâtiments des Oblats qui succédèrent aux Tiercelins après un long épisode de vacance débuté avec la Révolution. Ce lieu croise les savoirs, la culture écologique et les dimensions festives et didactiques indispensables à l'animation du site.

Cette ambition réanime la flamme du merveilleux et de collectif qui a fait vibrer la colline tout en s'adaptant à notre temps. Ainsi, Sion devient lieu de réflexion à propos 
des temps de l'écologie et de la défense de la biodiversité. S'il est dommage que le poirier pluriséculaire signalé par Maurice Barrès comme arbre " penderet » (servant de gibet) ait été détruit par un feu de friche vers 1980, de nombreux efforts s'ajoutent pour valoriser le territoire animé par la cité des Paysages. L'étroitesse des finages, l'intrication des parcelles de vergers ont évité à plusieurs communes de réaliser le remembrement. Aussi, encore aujourd'hui, les paysages attestent du maintien d'une agriculture qui n'est pas partout exclusivement productiviste et laisse la place à d'autres types de productions Cette remarque est à rapprocher de tout ce qui a été entrepris dans le proche bassin de captage des eaux de Vittel. Afin de conserver toutes les qualités des ressources en eau, les STH sont préservées, les épandages régulés, le maïs prohibé. Ces cadrages sont contractualisés et donnent lieu à des compensations financières.

Document 6 « Paysagissons!»

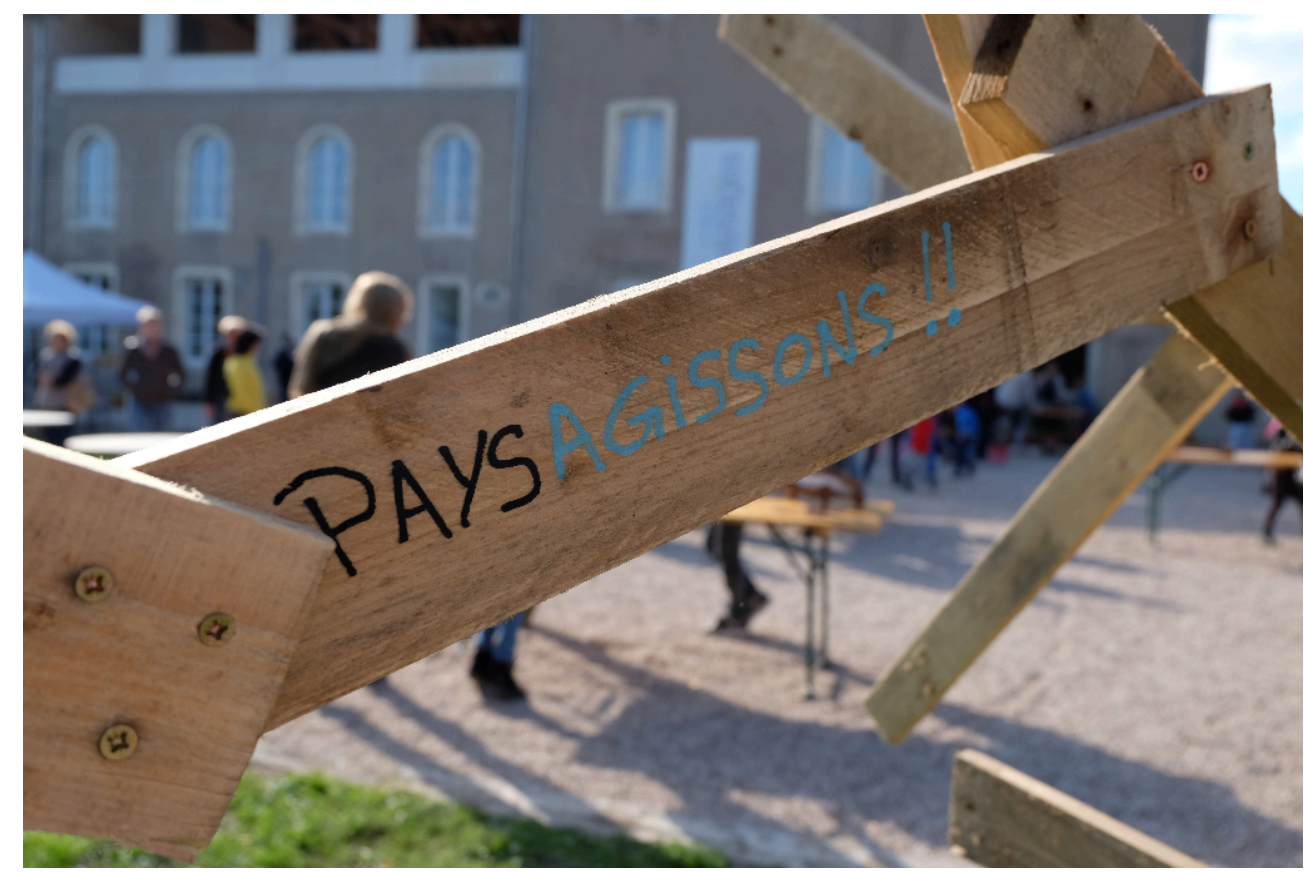

Un slogan en forme de néologisme inscrit sur une structure en bois brut. En arrière-plan, l'actuelle Cité des paysages qui réinvestit la résidence laissée par le départ des Oblats de Marie. Arrivée en 1856, cette congrégation avait, à l'époque de l'Empire colonial, envoyé des missionnaires de par la planète. Cet essaimage explique la présence passée de nombreux objets exotiques conservés et présentés dans leurs locaux.

Source : cliché Fl. Horny, 2016.

Dans sa thèse qui étudiait le découpage agraire du bassin du Madon, Rachel Jacopin a constaté plein de similitudes avec le Saintois autour de Sion. En utilisant la méthode des UAP (unités agro-physionomiques) chère à Jean-Pierre Deffontaines et Pascal Thinon, elle démontrait que les mosaïques agraires locales sont encore riches, complexes, ayant préservé des trames vertes et bleues. Les finages du Saintois sont peu étendus et conservent sur leurs marges des bribes de massifs forestiers afin de satisfaire les affouages. Cette remarque n'est pas une digression car elle permet de comprendre le potentiel de biodiversité local et la possibilité d'intégrer la qualité du territoire (Dedeire, 2018) comme ressource au prisme de la reconnaissance du site. L'échéancier des travaux entrepris (basilique, maison et ferme des Oblats) a été 
compliqué par une sorte d'avatar vite tourné en avantage. Il s'agit de la présence dans les toitures de la mairie et du couvent de trois espèces de chauves-souris bénéficiant d'une protection de rang européen : le Petit et le Grand Rhinolophe (classé d'intérêt communautaire), le Grand Mutin. Le grenier de la mairie de Saxon-Sion et les toitures de la ferme de l'ancien couvent hébergent chacune 150 Petits rhinolophes et ont été aménagés en « nursery " pour protéger cette espèce rare. Cette présence était occasion à relancer le récit à propos du site, l'animal nocturne ayant une aura particulière. Les chauves-souris disputent désormais la dimension merveilleuse aux seules "étoiles». Biodiversité, paysages amènes, paysage mis dans l'assiette pour parler des circuits courts et de commerce équitable s'inscrivent dans le sillon des rhinolophes. Ces sujets d'actualité sont à rapprocher de tous les questionnements sur les transitions. Les rhinolophes invitent à protéger le territoire traduit en habitat, en exprimant de la sympathie pour une espèce qui, il n'y a pas si longtemps était détestée, clouée aux portes, victime d'une superstition très injustifiée. Le CD 54 assure la présidence du comité de pilotage du site Natura 2000 appelé «Gites à chiroptères de la colline inspirée, érablières et pelouses de Vandeléville». Ceci est complété par plusieurs accords actés à l'échelon communal. En 2012, Saxon-Sion signe sa charte Natura 2000. Vaudémont confie au CD54 la gestion de 17 ha de pelouses xériques exploitées de façon extensive (fauchage tardif et faible charge en UPB/ha.).

Sur le site de pèlerinage de Sion, la longue durée de la dévotion à la Vierge fut toujours inscrite dans un processus dual. D'abord la proximité avec les dynasties ducales, ensuite l'articulation entretenue entre la foi et la nation. Actuellement le pèlerinage tente de s'associer à la promotion de la qualité des lieux. Ces évolutions révèlent des déroulés temporels discontinus, heurtés, à éclipses. La reconnaissance du site rebondit chaque fois qu'une nouvelle opportunité se dessine pour ciseler et faire évoluer le couple récit/territoire en activant des formes inédites de merveilleux ajoutées aux couches précédentes sans les effacer. Les relais du sacré et du merveilleux sont multiples pour faire vivre le lieu. Une approche de géographie historique est un éclairage utile pour aborder ce sujet plein de sensibilité, d'émotion, de subjectivité à propos d'une acropole où souffle l'esprit. Comme nous l'a appris Mircéa Eliade, Sion entre bien dans la catégorie des espaces sacrés par la majesté du site géographique, l'inscription sur une très longue courbe du temps et encore la dimension cosmique et tellurique qui anime le lieu.

\section{BIBLIOGRAPHIE}

Andriot Cédric, 2006, « Les Tiercelins à Sion (1626-1792) au service de l'Etat ». Nancy, Annales de l'Est, 2, p. 49-67.

Barral Pierre, 1991, L'esprit lorrain, cet accent singulier du patriotisme français. Nancy, PUN, 192 p.

Bertho Raphaël, Conésa Héloïse (dir.), Paysages français. Une aventure photographique 1984-2017.

Paris, BNF éditions, 2017, 292 p. 
Corajoud Michel, 1995, «Le paysage, c'est l'endroit où le ciel et la terre se touchent » Dans Roger Alain (dir.) La théorie du paysage en France », Seyssel, Champ Vallon, 462 p.

Debarbieux Bernard, 1995 « Le lieu, le territoire et trois figures de rhétorique ». Paris, L'Espace géographique, 2, p. 97-112.

Dedeire Marc, 2018, Aménagement rural et qualification territoriale. Bruxelles, PIE Peter Lang, col. Intégration régionale et cohésion sociale, $267 \mathrm{p}$.

Deffontaines Jean-Pierre, Thinon Pascal, 2001, « Les entités spatiales significatives pour l'activité agricole et pour les enjeux environnementaux et paysagers : contribution à une agronomie du territoire ». Paris, Les cahiers de l'environnement de l'INRA, 44, p. 12-38.

Eliade Mircéa, 1987, Le sacré et le profane, Paris, Gallimard, col. Poche, 185 p.

Fulaine Jean-Charles, 1997, Le duc Charles IV de Lorraine et son armée (1624-1675). Metz, éd. Serpenoise, $310 \mathrm{p}$.

Griselin Madeleine, d'Andelarrot Marcelle, Salvador Manola, 2017, Carnet de chemin de Besançon à Compostelle. Paris, Créer, col. Aquarelles, 87 p.

Husson Jean-Pierre, 2019 « Artialisation ». Publictionnaire. Mis en ligne le 10 mai 2019 http:// publictionnaire.huma-num.fr/notice/artialisation/

Jacopin Rachel, 2011, Les paysages construits par les agriculteurs de l'Ouest vosgien, déterminants et conséquences. Nancy 2, thèse de géographie, 15 décembre 2011, 460 p.

Jacquemin André, 1962, « En illustrant la colline inspirée de Maurice Barrès ». Nancy, Le Pays Lorrain, $\mathrm{n}^{\circ} 3$.

Jalabert Laurent, 2014, « Du territoire d'entre-deux à la limite : l'espace lorrain à l'épreuve de l'Etat, XVI ${ }^{\mathrm{e}}$-XVIII' ${ }^{\mathrm{e}}$ siècle », Revue de géographie historique, $\mathrm{n}^{\circ} 4$, http://rgh.univ-loraine.fr/artcles/ view/

Le Pays Lorrain, 1962, 3, Numéro thématique traitant du cinquantième anniversaire de la parution du roman de Barrès « La colline inspirée »; p. 77-133.

Le Tacon François, 2012, La croix de Lorraine: Du Golgotha à la France Libre. Metz, Serpenoise, 176 p. Louis René, 1990, « La colline inspirée, haut lieu. Une quête de racines, de sacré, de symboles ». Dans Crépu M., Figuier R. (direction), Paris, Autrement, p. 33-38.

Martin Philippe, 1995, Les chemins du sacré. Paroisses, processions, pèlerinages en Lorraine du XVI au XIX ${ }^{e}$ siècle. Metz, Serpenoise, 358 p.

Martin Philippe, 2002, Une guerre de Trente Ans en Lorraine : 1631-1661. Metz, éd. Serpenoise, 383 p. Martin Philippe, 2003, « La colline inspirée : lieu d'une mémoire lorraine nationale ou européenne ? » Dans Martin Ph., Roth F. (dir.) Mémoire et lieux de mémoire en Lorraine, Sarreguemines, Pierron, 357 p., p. 235-248.

Martin Philippe, 2004, «Les répits en Lorraine», Nancy, Mémoires de l'Académie de Stanislas, t XVIII, p. 531-544.

Martin Philippe, 2006, « Prier à Sion aujourd'hui ». Nancy, Annales de l'Est, 2, p. 210-229.

Olivier Laurent, 2006, « Le site de Sion avant Sion : archéologie de la colline inspirée ». Nancy, Annales de l'Est, 2, p. 19-46. 
Peltre Jean, 1995, «L'espace sacré des villages lorrains ».Dans Géographie historique et culturelle de l'Europe. Hommage au professeur X. de Planhol (textes réunis par J.-R. Pitte), Paris, PUS, 423 p., p. 308-316.

Pitte jean-Robert, 2005, « La géographie historique au service des problèmes d'aujourd'hui ». Dans Boulanger Philippe, Trochet Jean-René (dir.) Où en est la géographie historique ? Paris L'Harmattan, 346 p, p. 195-202.

Rufin Jean-Christophe, 2014, Immortelle randonnée. Compostelle malgré soi. Paris, Folio, 288 p.

Sgard Anne, 2008, « Entre rétrospective et prospective. Comment reconstruire le récit du territoire? ». Espace-Temps net, Travaux, 2008. URL : //www.espacestemps.net/articles/entreretrospective-etperspective/

Surdel Alain-Julien, 2006, « De Pierrepont à Sion : la Lanterne des Morts dans la commémoration lorraine après 1918 ». Nancy, Annales de l'Est, 2, p. 187-208.

Taveneaux René, 2000, « Barrès et la Lorraine ». Nancy, Le Pays Lorrain, 3, p. 219-225.

Thull Jean-François, 2006, « La colline de Sion-Vaudémont dans l'imaginaire lorrain ». Nancy, Annales de l'Est, 2, p. 167-186.

Wadier Roger, 1991, Les mirabelles, une aventure lorraine. Sarreguemines, Pierron, $127 \mathrm{p}$

Wagner Pierre-Edouard (textes réunis), 2003, Les Naudin entre Meuse et Vosges. Catalogue de l'exposition faite à la médiathèque du Pontifroy. Metz, Pontifroy, 115 p. + atlas.

\section{NOTES}

1. Entre 1600 et 1670, 570 miracles sont comptabilisés en Lorraine (Martin, 2004), pour l'essentiel sur les trois sites de N.-D. d'Avioth (173), Saint-Nicolas-de-Port (135) et N.-D. de Benoite-Vaux (111), soit 72,8\% des déclarations. Sion arrive ensuite avec 41 miracles répertoriés.

2. En 1477, c'est sous la protection de la bannière de N.-D. de Sion que le duc René II (1451-1519) fut le vainqueur de Charles le Téméraire (1477). Bibliophile et mécène, ce prince de la Renaissance fut le protecteur de l'école de cosmographie de Saint-Dié. Dès 1507, Waldseemüller devine que les découvertes faites dans le nouveau monde forment un tout. Le chanoine Vautrin Lud fait imprimer les lettres d'Amerigo Vespucci.

\section{RÉSUMÉS}

Sion est un haut-lieu lorrain. La "colline inspirée » assume trois fonctions successives. C'est d'abord un site de pèlerinage, de dévotion à Marie. L'endroit fut également au service d'ambitions politiques régionales (XVI-XVIII siècles) puis nationales (1873-1973). Aborder Sion sur le temps long permet d'étudier ces successions qui se chevauchent et modèlent le lieu pour arriver jusqu'à aujourd'hui. Restant un espace sacré chargé d'histoire, Sion devient un lieu où l'on réfléchit à propos de l'écologie des paysages. Ainsi se dessine une nouvelle page du récit de ce territoire sacré. 
Sion is a hot spot in Lorraine (France). The "inspired hill" has three successive functions. First of all, it is a pilgrimage site devoted to Mary. The place was also leveraged for regional (16-18th centuries) then national (1873-1973) political purposes. Observing Sion for long periods of time allows us to study these overlapping successions that shaped the site into its current state. Still a major sacred space full of history, Sion becomes a place where one thinks about the ecology of landscapes. Thus, a new page of the story of this sacred territory emerges.

\section{INDEX}

Keywords : Sion, Pilgrimage, marian devotion, nationalism, Maurice Barrès, artialisation, landscape ecology

Mots-clés : Sion, Pèlerinage, dévotion mariale, nationalisme, Maurice Barrès, artialisation, écologie des paysages

\section{AUTEUR}

\section{JEAN-PIERRE HUSSON}

Professeur émérite, Université de Lorraine, EA 7304, LOTERR 